\title{
Trichrome Mallory's stain may indicate differential rates of RNA synthesis in eutopic and ectopic endometrium
}

\author{
Maria Wołuń-Cholewa', Krzysztof Szymanowski², Mirosław Andrusiewicz', \\ Anna Szczerba ${ }^{1}$ and Jerzy B. Warchoł ${ }^{1}$ \\ ${ }^{1}$ Department of Cell Biology, K. Marcinkowski University of Medical Sciences, Poznań, Poland \\ ${ }^{2}$ Department of Mother's and Child's Health, K. Marcinkowski University of Medical Sciences, Poznań, \\ Poland
}

\begin{abstract}
Mallory's triple staining is a histochemical technique used mainly for analysing connective tissues and glands and other tissues. We have described the differences in the nuclear staining between eutopic and ectopic endometrium as well as endometrial hyperplasia and adenocarcinoma using the Mallory's method. The ultrastructural differences between eutopic and ectopic endometrium have been detected. In normal and hyperplastic endometrium the presence of stromal cell nuclei with an increased affinity to aniline blue has been observed. The affinity has disappeared after digestion of tissues with RNase. In cases of endometriosis, independently of cell types, the nuclei have shown affinity to orange G. Similar effects in adenocarcinoma have been noted. The ultrastructural studies have shown that in normal endometrium the stroma contained cells with euchromatic and low electron density cell nuclei. In endometriosis heterochromatic cell nuclei present both in the stroma and within glands have been detected. The results indicate that the Mallory's technique may be a useful tool for recognizing the differences between eutopic and ectopic endometrium. The affinity for aniline blue in normal and hyperplastic endometrium occurs most likely due to increased RNA synthesis. Based on Mallory's staining a similarity between hyperplasia and unchanged endometrium in contrast to similar results of the staining obtained in cases of adenocarcinoma and endometriosis may be suggested.
\end{abstract}

Key words: trichrome Mallory's stain, aniline blue, endometriosis, ultrastructure

\section{Introduction}

Endometriosis (ectopic endometrium) is commonly encountered in women during the sexual maturity. It affects $3-10 \%$ women of reproductive age. Among infertile patients and women with pain within pelvis the disease is even more frequent and its found in 20$90 \%$ of cases $[1,2]$.

Endometriosis is a presence of endometrial epithelium and stroma outside the uterine cavity. Most frequently it is detected within pelvis, first in ovaries and in pelvic visceral peritoneum. Similarly to typical endometrium, endometriotic foci demonstrate cyclic changes with bleeding in response to hormonal stimulation [2].

Correspondence: M. Wołuń-Cholewa, Dept. of Cell Biology, University of Medical Sciences, Rokietnicka 5D, 60-806 Poznań, Poland; tel.: (+4861) 8547186; fax: (+4861) 8547169,

e-mail: doskon@amp.edu.pl
In clinical practice, endometriosis is diagnosed using laparoscopy or laparotomy [2] together with biopsy and subsequent confirmation of typical lesions by histopathological examination.

The histochemical techniques use the selective chromophilic properties of cells for differential diagnosis. The applied acidic and alkaline histochemical stains allow to distinguish between basophilic, acidophilic or chromophobic cells. In several cases affinity of each stain and principles of cells staining have not been sufficiently recognised. However, methods of non-specific staining [3] may provide a useful diagnostic tool for recognition of pathological alterations, leading to differentiate them from normal tissues.

Methods, which allow to differentiate secretory cells, involved techniques of connective tissue fibres detections. The Mallory's stain belongs to the commonly used techniques in which three dyes: aniline blue, orange $\mathrm{G}$ and acid fuchsin are combined [4,5]. This multiple-dye staining has a capability of differen- 
tiating a large number of tissues structures. Collagen fibres are shown in dark blue, collagen-containing connective tissue in blue, erythrocytes in orange, and chromatin, nuclei, basophilic cytoplasm, and muscle cell cytoplasm are visible in red colour. The Mallory's staining is currently used for detection of many tissue components in several organs $[4,5]$.

In this paper we describe a difference in the nuclear staining between eutopic end ectopic endometrium using Mallory's triple-chrome method. The results obtained from Mallory's staining were compared to results noted in cases of endometrial hyperplasia and adenocarcinoma. Moreover, the ultrastructural differences between eutopic and ectopic endometrium were confirmed using a transmission electron microscope.

\section{Materials and methods}

Collection and preparation of endometrial samples. Tissue samples of normal endometria were obtained during hysterectomy from 8 women diagnosed with cervical intra-epithelial neoplasia. The fragments of ovarian endometriosis from 15 women (age: 28-42 years, median: 35) were collected during laparoscopy or laparotomy with lesion biopsy. The diagnosis of endometriosis was confirmed by histopathological examination of the tissue. All the examined women (with or without endometriosis) had regular menstrual cycles and experienced no hormonal treatment for at least 6 months before surgery. The specimens were obtained during the secretory phase of the menstrual cycle, confirmed by serum hormone level measurement. Surgical tissue specimens were obtained at the Department of Mother's and Child's Health, Poznan University of Medical Sciences. Collected tissue samples were washed in calcium-free PBS to remove blood residue, and incubated in serum-free DMEM/Ham's F-12 culture medium (Sigma, St. Louis, USA). Several tissue samples were fixed in $4 \%$ buffered paraformaldehyde (PFA), pH 7.4 (Sigma), processed and paraffin-embedded.

In addition, archival paraffin-embedded tissue sections of ovarian endometriosis $(n=15)$, endometrial adenocarcinoma $(n=4)$ and endometrial hyperplasia without atypia $(n=1)$ were used for Mallory's trichrome staining (age of patients: 32-45 years, median 35). Before Mallory's trichrome staining, serial sections were dewaxed in xylene and rehydrated in a graded ethanol series.

Mallory trichrome staining. Samples of eutopic and ectopic endometrium, fixed in 4\% PFA and processed as described above were cut in sections of two microns. Tissue sections after dewaxing and rehydrating were stained according to the Mallory's trichrome procedures. The staining was carried out in two solutions. At first, the tissue sections were stained in $1 \%$ acid fuchsin (Sigma) solution in distilled water. The second step included counterstaining in a solution of $0.5 \%$ aniline blue (Sigma), $2 \%$ orange $\mathrm{G}$ (Sigma), and $2 \%$ oxalic acid (Sigma) in distilled water. The standard steps followed those described by Bloom and Fawcett [3].

Enzymatic digestion. Before staining sections were incubated for 5 hrs at $37^{\circ} \mathrm{C}$ with $0.4 \mathrm{mg} / \mathrm{ml}$ RNAse A (Sigma) in distilled water $\mathrm{pH}$ 6.8 or in $0.2 \mathrm{mg} / \mathrm{ml}$ DNAse II (Sigma) in $0.03 \mathrm{M}$ phosphate buffer $\mathrm{pH} 7.0$, activated with $0.06 \%$ magnesium chloride or $0.1 \%$ trypsin (Sigma) in 0.01 phosphate buffer $\mathrm{pH}$ 7.6. Adjacent control sections were incubated in the phosphate buffer without the enzymes 4 .

Ethical issues. The study was approved by the local Ethics Review Board. All the patients participating in the study provided their written consent.
Statistical analysis. Chi-square test (STATISTICA ver.5 software, Statsoft, Krakow, Poland) was used to compare ratio of stained with aniline blue to non-stained cell nuclei of eutopic and ectopic endometria. $\mathrm{P}$ value $<0.05$ was considered as significant.

Electron microscopy. For electron microscopy analysis, tissue specimens of eutopic and ectopic endometrium were immersed at $20^{\circ} \mathrm{C}$ for at least $2 \mathrm{hrs}$, in a fixative solution containing $8 \%$ glutaraldehyde (Taab, Berkshire, UK) and $8 \%$ paraformaldehyde (Merck, Darmstadt, Germany) buffered with $0.1 \mathrm{M}$ phosphate buffer (Merck), pH 7.3. After immersion, specimens were cut and incubated overnight in fixative solution, rinsed for $60 \mathrm{~min}$ in $0.1 \mathrm{M}$ phosphate buffer, $\mathrm{pH} 7.3$, and postfixed in $2 \% \mathrm{OsO}_{4}$ in $0.1 \mathrm{M}$ phosphate buffer for 2 hrs (Merck). The specimens were dehydrated in ethanol, block-stained with alcoholic uranyl acetate and embedded in Spurr's medium (Merck). After contrasting, the ultrastructure of epithelial cells and fibroblasts were examined using JEOL 100 Transmission Electron Microscope [6].

\section{Results}

The Mallory's trichrome staining of eutopic and ectopic endometrium cell nuclei demonstrated a variable affinity to the applied dyes. Cell nuclei in normal endometrium manifested strong affinity to aniline blue $(77 \% \pm 11$ blue cell nuclei) (Fig.1a), which was particularly well visible in stromal cells. Whereas, cell nuclei of epithelial cells demonstrated typical for this technique affinity to orange G (Fig. 1a, orange cell nuclei). Nevertheless, within all glands of normal endometrium only a few epithelial cell nuclei were stained with aniline blue (Fig. 1a). In cases of ectopic endometrium, the application of Mallory's technique demonstrated the presence of both orange and blue cell nuclei in glands $(66 \% \pm 7$ and $34 \% \pm 5$ respectively) as well as in the stroma $(59 \% \pm 8$ and $41 \% \pm 5$ respectively; Fig. 1b). Statistical analysis of the obtained results for glands and stroma cells indicated significant differences in the number of blue stained cell nuclei in eutopic and ectopic endometria. For each cell type $p$ value was less than 0.05 .

At subsequent stages of the studies, the paraffin sections were enzymatically digested with ribonuclease A, deoxyribonuclease II and trypsin. After digestion with ribonuclease cell nuclei of the analysed material completely lost their affinity to aniline blue. All cells nuclei, regardless of type, were stained with orange G (Fig. 1c). Pre-treatment with DNase and trypsin did not induce any changes in the cell nuclei staining.

Afterwards we decided to examine effects of Mallory's staining in cases of endometrial hypertrophy and endometrial carcinoma. In endometrial hypertrophy cell nuclei were found to exhibit strong affinity to aniline blue (Fig. 1d). Staining effect was independent of the stained cell type. Whereas in endometrial carcinoma, nuclei of epithelial cells manifested strong affinity to orange $\mathrm{G}$, while cell nuclei of stromal cells were stained both with orange $\mathrm{G}$ and aniline blue (Fig. 1e).

Ultrastructural studies on eutopic and ectopic endometria resulted from differences in Mallory's 

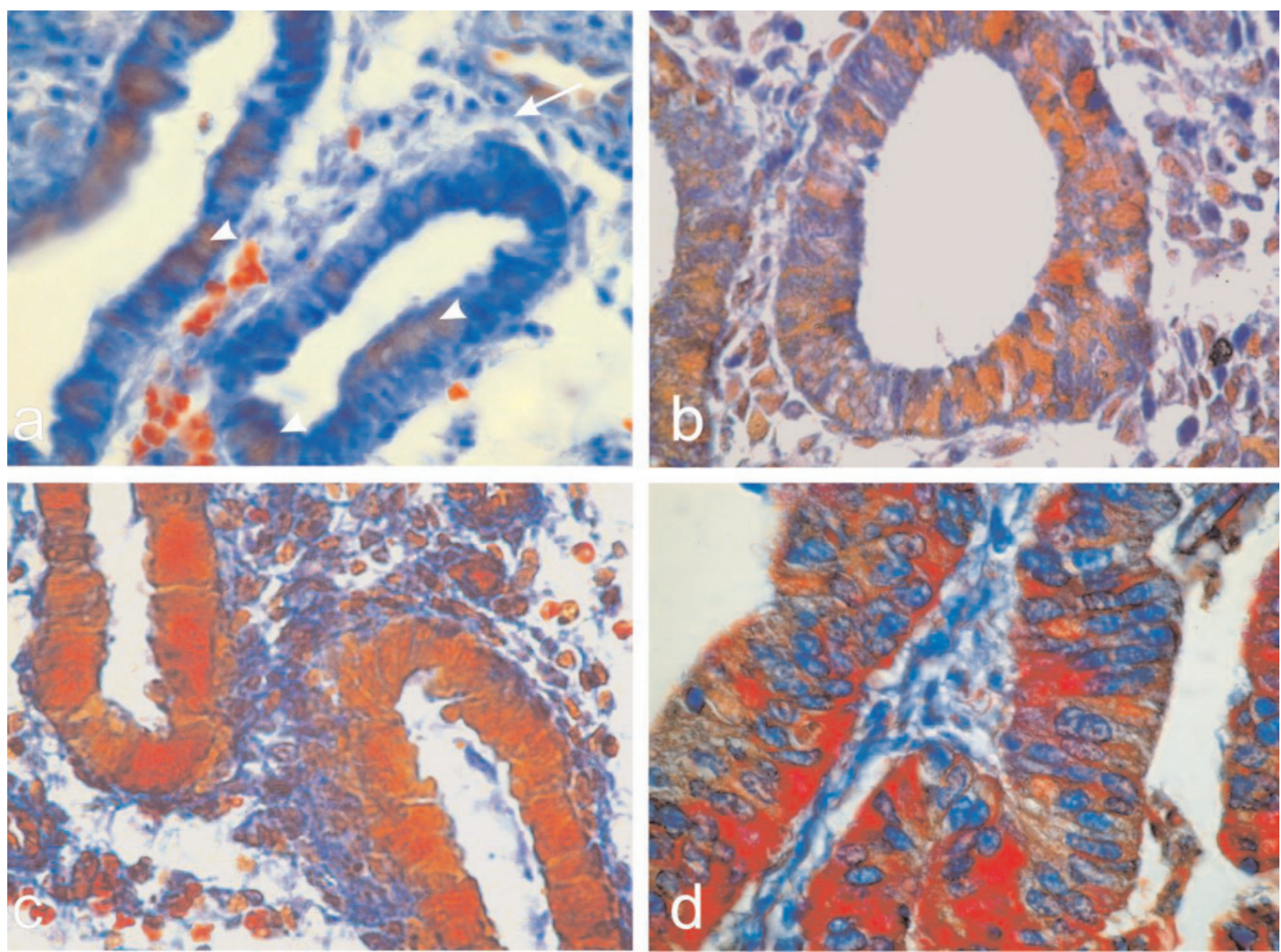

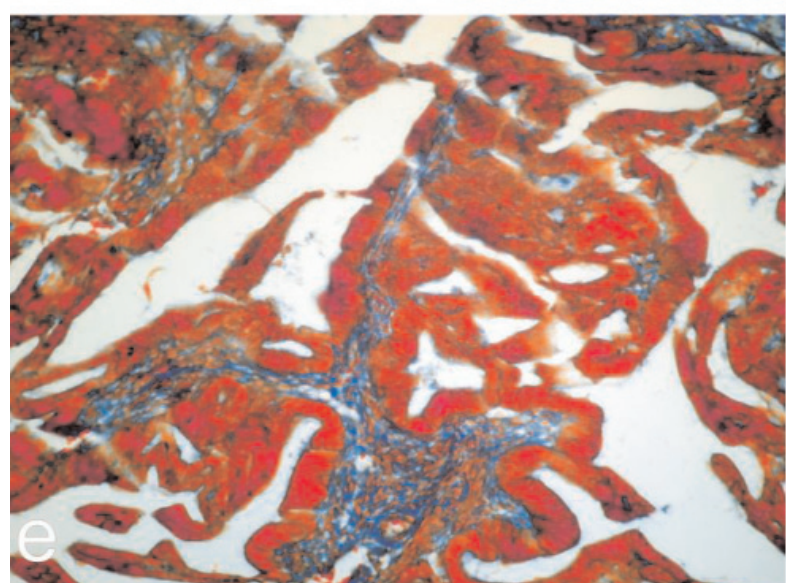

trichrome staining. In normal endometrium the stroma contained cells with euchromatic and low electron density cell nuclei (Fig. 2a and 2b). Most epithelial cells have an elevated nucleocytoplasmic ratio and nuclei with high amount of heterochromatin. In endometriosis heterochromatic cell nuclei were present both in the stroma and glands and had an irregular shape (Fig. 2c and 2d).

\section{Discussion}

In several publications [7-9] similarities in histological structure of endometrium located out of uterine mucosa
Fig. 1. The Mallory's trichrome staining of endometrium (a) eutopic without RNase A, (b) ectopic, (c) eutopic RNase treated, (d) hyperplasia of endometrium without atypia and (e) endometrial carcinoma. Cell nuclei in normal endometrium and stromal cells manifested affinity to aniline blue. Some of epithelial cells nuclei demonstrated affinity to orange $\mathrm{G}$ (indicated by arrowheads). In ectopic endometrium, the presence of both orange and blue cell nuclei in the glands as well as in the stroma was observed. In case of digestion with ribonuclease A all nuclei stained with orange $\mathrm{G}$. In endometrial hyperplasia without atypia cell nuclei exhibit affinity to aniline blue. Endometrial carcinoma nuclei of epithelial cells manifested strong affinity only to orange $\mathrm{G}$ while cell nuclei of stromal cells were stained both with orange $G$ and aniline blue.

and in endometrium of normal localization have been documented, on the contrary to some clinical cases, where ectopic glandular epithelium differ in function and morphology from normal endometrial cells. Most frequently described anomalies have been explained by the endometriosis early development or by a mechanism leading to endometriosis. Age of the endometriosisaffected women has also been found to be important, what was is confirmed by structural and functional identity of endometrium and the ectopic glandular epithelium $[1,9,10]$.

There are no publications describing suitability of histochemical techniques which might provide a use- 

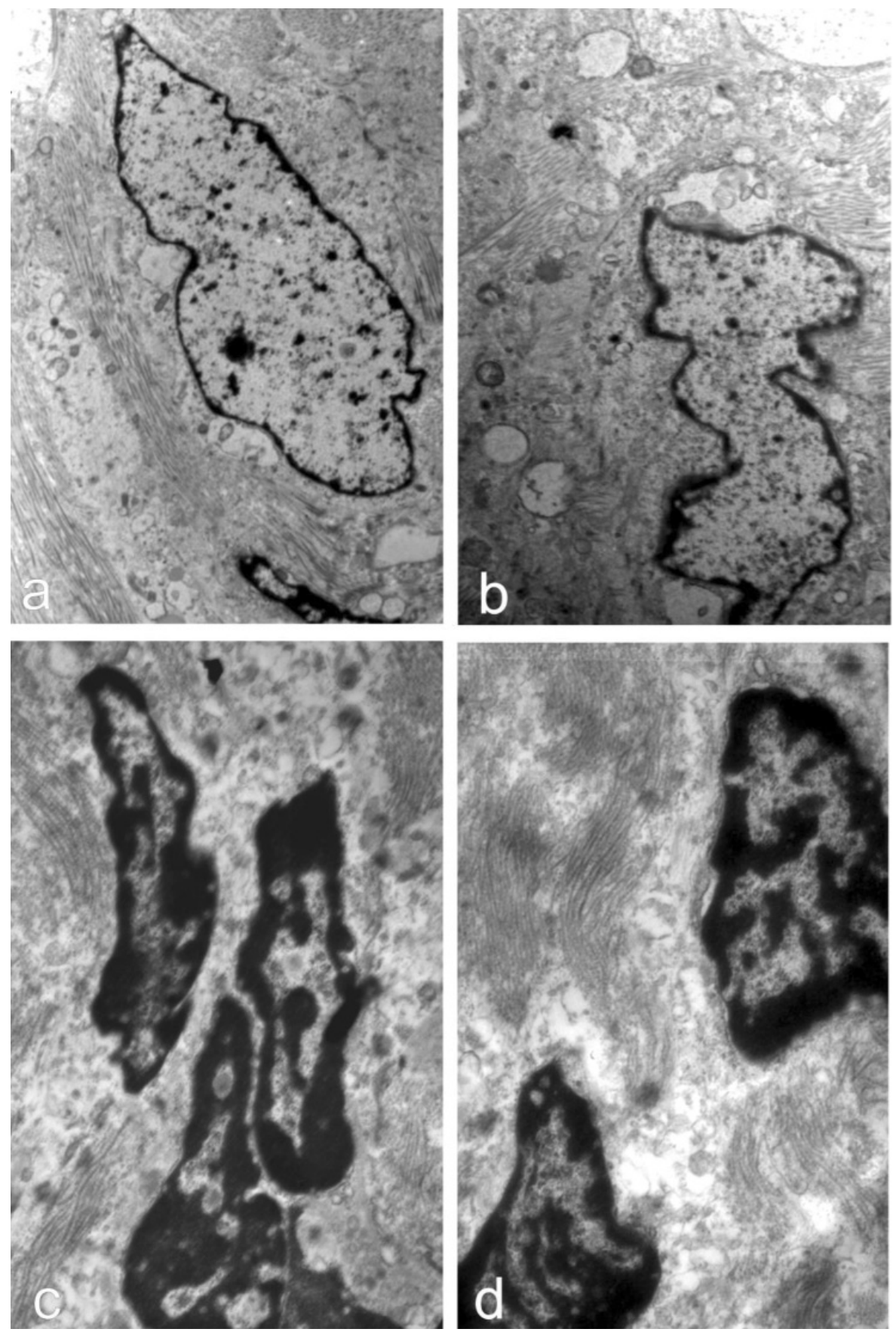

Fig. 2. The nuclei ultrastructure in $(\mathbf{a}, \mathbf{b})$ eutopic and (c, d) ectopic endometria. In the case of normal endometrium the stroma contained cells with euchromatic and low electron density cell nuclei with high amount heterochromatin. In endometriosis heterochromatic cell nuclei have been present both in the stroma and within glands. These cell nuclei had an irregular shape. ful tool for diagnosis of pathological alterations in endometriosis. The methods applied now are based on analysis of differences in expression level of selected genes among the normal and pathological tissue. There is a need for novel approach to distinguish eutopic and ectopic endometrium. The usage of CA125 level as a specific marker of endometriosis has regrettably not been proven yet. Such studies require a significant financial support and are very time-consuming [11].

In these studies we have decided to apply the classical trichrome staining method according to Mallory in cases of eutopic and ectopic endometrium. Consistent with the effects of Mallory's staining, secretory cells nuclei have been stained with the orange $G[4,5]$. Moreover, cell nuclei exhibited strong affinity to aniline blue. The obtained results manifested certain consistency with the previously obtained. Kovaks and coworkers [12] applied a technique of trichrome staining defined as astrin, similar to Mallory's method. In their studies differences in the originally assumed staining pattern in cell nuclei of liver and pancreas were noted. Those are dependent on the form of nuclear chromatin. Moreover, authors discovered that stimulation of cells with pilocarpine or prednisolone resulted in stronger blue staining of cell nuclei. Next experiments with enzymatic digestion of studied tissues suggested that 
staining differences reflected variable content of DNA and protein in cell nuclei and a protein-dependent change in DNA structure [12]. Delgado and co-workers [13] found also that differences in staining of cell nuclei with acidic aniline blue reflected in disturbed membrane integrity.

In our studies presence of cell nuclei with an increased affinity to aniline blue has been observed mostly in stroma of a normal endometrium. In contrast to that, only a few epithelial cells have manifested affinity to aniline blue and have been stained typically for Mallory's technique, as well as in case of hypertrophic endometrium. In endometriosis, both in freshly embedded material and in archival samples, an increased number of cells regardless of type with an affinity to orange $\mathrm{G}$ have been detected which was similar to results from adenocarcinoma. Observations suggest a similarity between tissues affected by a simple hypertrophy and an normal endometrium. What is more, adenocarcinoma and endometriosis, are similarly stained, which seems to point to alike character of the two tissue types.

In this research effects of enzymatic digestion with three enzymes $[4,5]$ has been conducted. Only ribonuclease A digestion has been found to affect chromophilic properties of cell nuclei in endometrium. After such a treatment all cell nuclei have been strongly stained with orange $\mathrm{G}$. This suggests that cells with cell nuclei stained blue manifest an increased synthesis of RNA. In order to prove those suggestions, staining with acridine orange was performed. It allows cell type distinguish depending on DNA and RNA presence [data not shown]. Similarly to Mallory's technique, differences in RNA contents have been detected and confirmed the dependance on the type of endometrium.

In order to clarify differences in the manner of staining of cell nuclei examined in this study, ultrastructural studies on normal endometrium and endometriosis have been performed. Eutopic endometrium has demonstrated an increased number of cells with euchromatic cell nuclei. In ectopic endometrium cells with electron-dense heterochromatin have been detected.

In order to explain the obtained results, cell nuclei condition at the moment of performing the experiments have been considered. Cell nuclei stained with aniline blue have been probably involved in transcription processes. The increased amount of RNA a effects absorption of cell structures and, in effect, the cell nuclei is stained in blue using the Mallory's technique. As a result cell nuclei are stained in different colour than expected.

Effects of staining and the resulting differences between eutopic and ectopic endometrium require further studies. It may be assumed that the reason of these results reflects presence in aniline blue molecule $-\mathrm{NH}_{2}$ and $-\mathrm{NH}+$ groups. In acidic environment $(\mathrm{pH}<3.0)$ they undergo protonization and, therefore, they can selectively bind to the newly synthesised negatively charged RNA. On the other hand, in the non-transcriptive phase anionic groups of nucleic acids are coupled to nuclear proteins and interact with orange G. Summing up, affinity of certain cell nuclei to orange $\mathrm{G}$ or aniline blue may be caused by an increased transcription [4,5].

Present studies have shown that classical histological techniques, forgotten nowadays, may provide an easy and economic way to distinguish cell nucleus transcriptional activity in normal and pathologically altered tissues which allows in parallel a rapid lesions diagnosis.

Acknowledgement: The study was supported in part by National Committee for Scientific Research, grants No.: NN 407084836 and NN 407109533.

\section{References}

[ 1] Bondza PK, Maheux R, Akoum A. Insights into endometriosis-associated endometrial dysfunctions: a review. Front Biosci (Elite Ed). 2009;1:415-28.

[2] Wolun-Cholewa M, Butowska W, Fischer N, Warchol W, Nowak-Markwitz E. 5-aminolevulinic acid-mediated photodynamic therapy of human endometriotic primary epithelial cells. Photomed Laser Surg. 2009;27:295-301.

[3] Bloom W and Fawcett Don W. A textbook of histology. 10th ed. Philadelphia, PA: W.B. Saunders; 1975.

[4] Chieffi-Baccari G, Marmorino C, Minucci S, Di Matteo L, d'Istria M. Mallory stain may indicate differential rates of RNA synthesis: II. Comparative observations in vertebrate nuclei. Eur J Histochem. 1992;36:187-96.

[5] Chieffi Baccari G, Marmorino C, Minucci S, Di Matteo L, Varriale B, d'Istria M, Chieffi G. Mallory stain may indicate differential rates of RNA synthesis: I. A seasonal cycle in the harderian gland of the green frog (Rana esculenta). Eur J Histochem. 1992;36:81-90.

[ 6] Wasko R, Wolun-Cholewa M, Bolko P, Kotwicka M. Effect of bromocriptine on cell apoptosis and proliferation in $\mathrm{GH} 3$ cell culture. Neuro Endocrinol Lett. 2004;25:223-8.

[ 7] Matthews CJ, Redfern CP, Hirst BH, Thomas EJ. Characterization of human purified epithelial and stromal cells from endometrium and endometriosis in tissue culture. Fertil Steril. 1992;57:990-7.

[8] Redwine DB. Was Sampson wrong? Fertil Steril. 2002;78:686-93.

[ 9] Vinatier D, Cosson M, Dufour P. Is endometriosis an endometrial disease? Eur J Obstet Gynecol Reprod Biol. 2000;91:113-25.

[10] Campbell IG \& Thomas EJ. Endometriosis: candidate genes. Hum Reprod. 2001;7:15-20.

[11] Winkel CA. Evaluation and management of women with endometriosis. Obstet Gynecol. 2003;102:397-408.

[12] Kovács P, Csaba G, Balogh G. Detection of different functional states of the cellular nucleus with a new trichrome staining technique. Acta Histochem. 1982;71:73-5.

[13] Delgado NM, Sánchez-Vázquez ML, Hernández O, Reyes R. Correlation between sperm membrane destabilization by heparin and aniline blue staining as membrane integrity index. Arch Androl. 1998;40:147-52.

Submitted: 3 August, 2009 Accepted after reviews: 17 January, 2010 